\title{
Thermomechanical coupling as a criterion of the yield point of spheroidal graphite cast iron
}

by SCHMIDT J.*

\author{
* Foundry Research Institute, 30 - 418 Cracow, Zakopianska 73, POLAND
}

\begin{abstract}
The determination of the critical levels of stresses in the form of the yield point for materials with quasiparabolic course of the tension curve is based on the conventional determination of remaining strain. The development of remaining strain, determined through observation of an increase in temperature on the surface of a specimen during the growth of stress $\sigma_{i i}$, confirmed by an increase in the values of the factors of acoustic emission, allows the physical determination of the place of occurrence of the yield point. The experiment was made for the spheroidal graphite cast iron, grade Zs 60002.
\end{abstract}

\section{Introduction}

The effects of the thermomechanical coupling in solid bodies caused by external application of mechanical loads depend on the structural state in which the body (metal) is being examined. According to the effects which the applied load causes, we can distinguish the following types of coupling: coupling through the change of volume $\Delta \mathrm{V} \rightarrow \Delta \mathrm{T}$; coupling through hysteresis of the changes caused by load; coupling resulting from the movement of structural defects.

In metals where the process of plastic deformation is the most distinct one, almost the whole work of external forces is changed into heat. That is why the period in which the metal becomes plastic can be related with a noticeable increase in temperature. The change of the thermodynamic state of the body caused by the loading process can be a physical signal of reaching the yield point, determined conventionally in the materials with quasiparabolic course of the tension curve as $R_{0,1}$ or $R_{0,2}$. To such materials spheroidal graphite cast iron can be included. To achieve the intended

determine the beginning of the rapid development of the ductile transition in a specimen, it was necessary to observe the thermal state in the examined volume of the specimen through observations of temperature on the specimen surface. Using the emission effect of infrared radiation, the change of temperature was measured by an AGA 680 apparatus. To obtain a high resolution of the measurement, a system of image analysis of the field of isotherms corresponding to the state of stress $\sigma_{i i}$ was developed, making a quantitative analysis of the images referred to a standard system. The digital image analyzer System III Analytical Measuring System was used [1].

Temperature measurement should be performed under quasiadiabatic conditions.

The finally obtained formula for determination of the temperature change is as follows:

$$
\Delta T=\frac{33.56}{I_{p}} \frac{2}{\varepsilon_{0}} A_{i} \Delta X
$$


http://dx.doi.org/10.21611/qirt.1994.015

where: $I_{\rho}$ - initial isotherm of measurement; $A_{i}$ - coefficient of calibration for the measuring conditions; $\Delta X$ - change in the field of isotherms read out from the analyzing system.

The suitable shape of the specimen was designed and the barriers of heat flow between the specimen and the special grips of the testing machine were applied (figure 1).
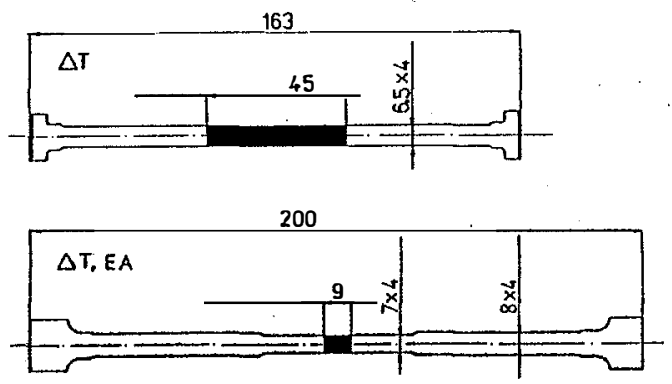

Fig.1. Geometry of the specimen; black area, indicates the fields examined in the test

The specimen was placed in the sheath reducing the interferences of the emission of the infrared radiation and of the air fluctuation. The axial strain on the specimen surface was measured by means of strain gauges stuck on the side opposite to the examined side of the specimen. The acoustic emission occurring during the loading cycle was recorded on an Acoustic Emission Technology Corporation apparatus, with simultaneous recording of temperature changes in the central part of the specimen. To describe the phenomenon of acoustic emission, the indicators of the mean voltage of the effective signal (EA RMS) and of the number of the events counting (EA IMP) were used.

The error of the analysis was estimated taking into account experimentally all the conditions of measurement for a constant level of saturation of the field of the examined isotherm. The values of standard deviation were obtained for the fields examined in the test:

$$
\Delta T \rightarrow \quad S= \pm 0,007 \mathrm{~K} \quad(\mathrm{~L}=45 \mathrm{~mm}) ; \quad \Delta \mathrm{T} \rightarrow \quad \mathrm{S}= \pm 0,009 \mathrm{~K} \quad(\mathrm{~L}=9 \mathrm{~mm})
$$

The values of change in temperature are given to the third decimal place which results from the mathematical formula of computations. .

\section{Material}

Tests were carried out on a perlitic-ferritic cast iron, grade Zs 60002, (Polish Standard). The properties of this cast iron where described in the complete tensile test with proof stresses determination:

$R_{m}=626$ - $640 \mathrm{Mpa} ; R_{0,02}=355 \mathrm{Mpa} ; R_{0,2}=464 \mathrm{MPa} ; R_{A}=330 \mathrm{MPa}$;

$E=184400 \mathrm{MPa} ; \mu=0.27 ; A_{5}=3.1-4.8 \%$

The specimens were loaded in single cycles at a rate of $\varepsilon=1.6 \times 10^{-3} \mathrm{~s}^{-1}$ (measurement of $\Delta T$ ) and at a rate of $\varepsilon=6.6 \times 10^{-4} \mathrm{~s}^{-1}$ (measurement of $\Delta T, E A$ ) and in monotonic mode at a rate of $\varepsilon=6.6 \times 10^{-4} \mathrm{~s}^{-1}$ (measurement of $\Delta T, E A$ ).

\section{Experimental}

The aim of the experiment was to investigate by means of thermal effect, observed on the surface of the specimen, the critical development of remaining strain. Figure 2 
http://dx.doi.org/10.21611/qirt.1994.015

represents the temperature change observed on the surfaces of the specimens during loading and unloading of cast iron.

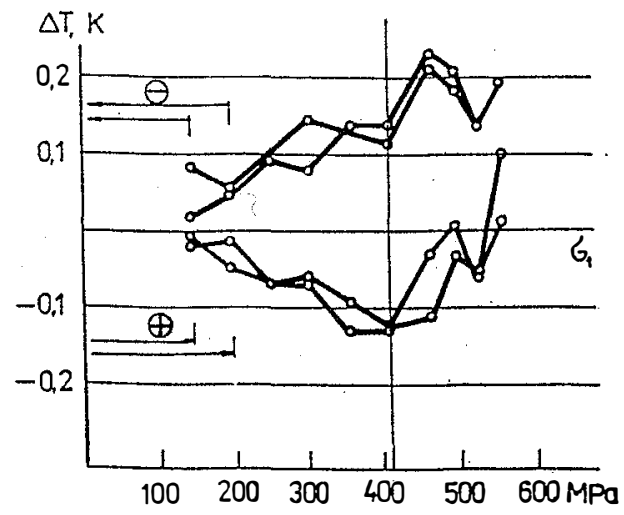

Fig.2. Temperature of two specimens $(\Delta T)$ for the state of loading and unloading $\varepsilon=1.6 \times 10^{-3} \mathrm{~s}^{-1}$

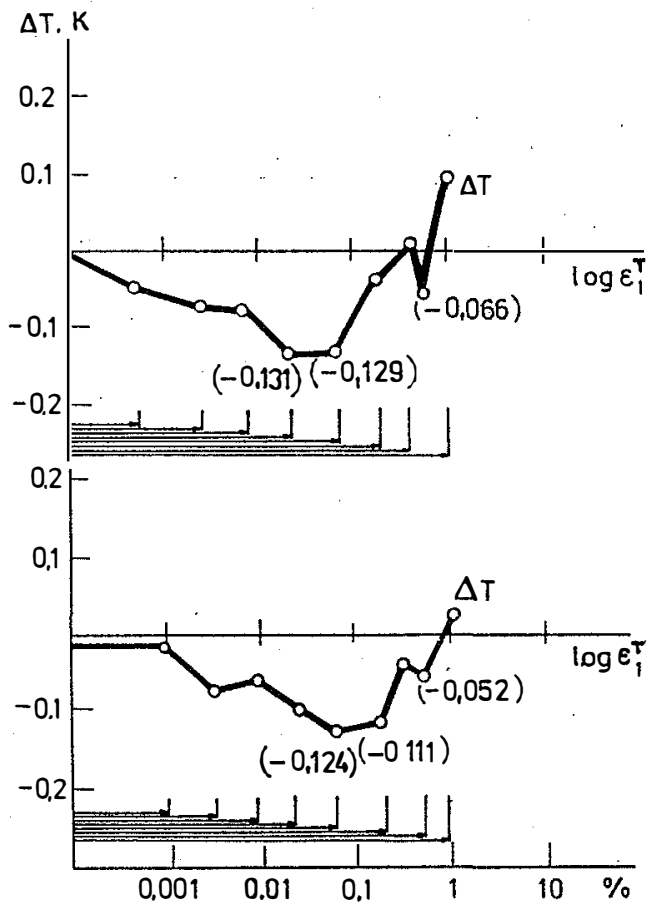

Fig. 3. Temperature of two specimens $(\Delta T)$ referred to the remaining strain $\left(\varepsilon^{\top}\right) ; \quad \varepsilon=1.6 \times 10^{-3} \mathrm{~s}^{-1}$

The absolute increase of temperature, corresponding to the same cycle at the loaded side and at the unloaded one within a range of small remaining strains, show similar values $(+\Delta \sigma \rightarrow-\Delta \mathrm{T})(-\Delta \sigma \rightarrow+\Delta \mathrm{T})$ for the level of loading of up to $410 \mathrm{MPa}$.

Figure 3 presents the temperature changes observed on the specimen surface during loading, referred to the value of remaining strain. The line of the temperature changes corresponding to the loaded side reveals after reaching the negative extremum a rapid temperature increase from the level $\varepsilon^{\top}<0.1 \%$. It is consistent with the beginning of distinct differences in absolute values of the temperature increases which correspond to both the loaded and unloaded side in the same cycle. The temperature increase is for a moment stopped at the level of $500-525 \mathrm{MPa}$ which is in conformity with $\varepsilon^{\top}=0.40$ $0.45 \%$. On the basis of the carried out microscopic observations it was stated that the momentary decrease in temperature, is related with the stopping of the slip lines at the ferrite grain boundary to the moment at which with further rise of stress they can be reactivated and can expand to the remaining phase interface and in the grains of perlite. Spheroidal graphite cast iron is a material which from the beginning of the loading cycle shows the existence of the remaining strains resulting from the heterogeneity of structure. The emission activity occurs even in the first cycles of loading in a range of negative increases of temperature caused by the changing volume of material. This proves the 
http://dx.doi.org/10.21611/qirt.1994.015

occurrence of an intensive process of dislocations and the formation of the sources of plastic strain, blocked in further course of loading. An obvious increase in the amplitude of signal $A E$ (RMS) has occurred from the stress level of $410 \mathrm{MPa}$ in the range when the surface of the examined specimen has reached the temperature of the initial condition (figure 4).

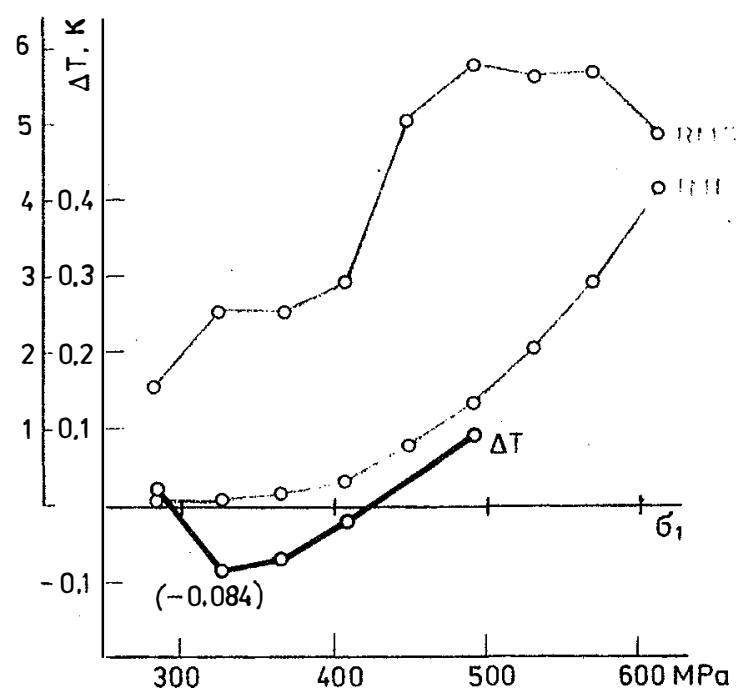

Fig.4. Temperature of specimen $(\Delta T)$ and acoustic emission (EA) referred to the stress $\left(\sigma_{1}\right) ; \varepsilon=6.6 \times 10^{-4} \mathrm{~s}^{-1}$

For the monotonic loading course, the maximum of the momentary effective value of the RMS signal occurred at the level of $460 \mathrm{MPa}$, but the quickest increase of this value in relation to the rise of stress has already begun from the value of $405 \mathrm{Mpa}(\sigma=405 \rightarrow$ $450 \mathrm{MPa}$ ). These changes are connected with the character of the temperature increases observed on the surface of the specimen (figure 5).

\section{Discussion}

The observation of temperature on the specimen surface gives a very clear image of the change in its inner state which is caused by the effect of the mechanical strain. This atrong observation can be specially useful when the effect of strain before the ductile transformation of specimen area is to be evaluated. The measurements of the temperature changes carried out in some selected spots by means of precise methods used for steel present the occurrence of an elastoplastic limit stress at the period of the negative temperature extremum [2-3]. For materials with a quasiparabolic course of the tension curve a criterion of the beginning of distinct plastic changes are $\sigma_{0,1}$ or $\sigma_{0,2}$, adopted according to the authors' opinion.

For spheroidal graphite cast iron the commonly accepted criterion is that of $R_{0,2}$. For the investigated spheroidal graphite cast iron the application of the criterion $R_{0,2}$ in the complete tensile test was equal to the stress of $460 \mathrm{MPa}$. In the test of monotonic tension it is equal to a maximum of the effective EA RMS signal and to the temperature of the 
specimen surface equal to the temperature of the initial state of the test. In the test of cyclic loading the stress values of $460 \mathrm{MPa}$ are equal to the levels $\varepsilon^{\top}=0.187 \% ; 0.189 \%$.

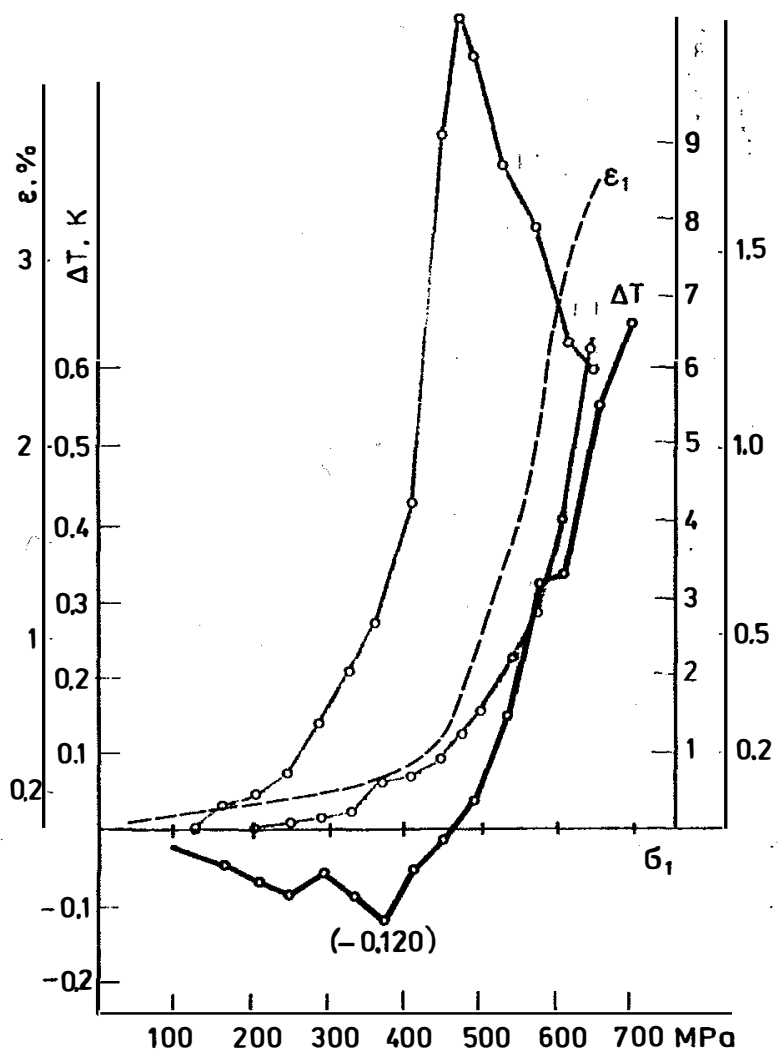

Fig.5. Temperature of specimen $(\Delta T)$ acoustic emission $(E A)$ and total strain $\left(\varepsilon_{1}\right)$ referred to the stress $\left(\sigma_{1}\right)$; monotonic loading curve

In terms of temperature this value is in the area of a quick drop in the negative temperature increase from the negative extreme value. Examining the increases in temperature for $\varepsilon=1.6 \times 10^{-3} \mathrm{~s}^{-1}$ it can be noted that the negative extremum of temperature occurs for the level of remaining strain $\varepsilon^{\top}=0.06 \rightarrow 0.07 \%$, which corresponds to a stress $\sigma_{1}=410 \mathrm{MPa}$. For test loading with the strain rate of $\varepsilon=6.6 \times$ $10^{-4} \mathrm{~s}^{-1}$, corresponding to the rate applied in standard tensile tests, the negative extremum occurs at a stress level of $\sigma_{1}=340-370 \mathrm{MPa}$. The differences result from the effect of strain hardening acting at higher strain rates.

At this level also occurs the period of the quickest rise of the signal EA RMS and a quicker rise in the number of events EA IMP. The lines of temperature changes which correspond to the period of the emission activity show incidentally the positive temperature increases on the surface of the specimen from the cycle level $\sigma_{1}=410$. $425 \mathrm{MPa}$. The value of the temperature increase in the specimen is a result of the 
http://dx.doi.org/10.21611/qirt.1994.015

superposition of the negative temperature increases which result from the effect of roversible strain and the positive temperature increases connected with heat dissipation.

From the physical point of view exceeding the region of maximum negative of temperature should be regarded as a starting point for the sources of remaining strain which ands in the ductile transformation of the whole region. In this range the growth in acoustic omission assumes the highest rate, too. So, the stress $\sigma_{1}$ corresponding to the exceeded ronge of remaining strain $0.1 \%$ should be regarded as critical in the process of loading perlitic-ferritic spheroidal graphite cast iron. Hence, from the point of view of the material characteristic, as a criterion of the yield point the stress $\sigma_{0,1}$ should be accepted.

Karamara [4] writing down the value of energy lost in the cycle of single loads of apheroidal graphite cast iron obtained for the first two levels of stress and for the level of $300 \mathrm{MPa}$ a momentary loss of energy used for the performance of the cycle. This is conistent with the momentary increase in temperature during both methods of loading for a lovel of $290 \mathrm{MPa}$. It seems that the decrease of the amount of absorbed heat is related with the mechanism of action of the geometrically indispensable dislocations, first described by Ashby [5].

The observations of the indications of the acoustic emission, and especially of the number of the counts. EA IMP, as well as the observations of temperature during the development of the field of stress at cyclic tension of spheroidal graphite cast iron confirm the hypothesis that the positive temperature changes observed on the surface of the specimen are caused by the process of internal friction.

\section{REFERENCES}

[1] SCHMIDT (J.). - Thermographic effect of the development of the stress field with the application for the analysis of the course and effects of cyclic loadings in foundry materials. Doctor's Thesis. Foundry Research Institute, Cracow 1988.

(2) SRODKA (W.) - Thermomechanical coupling in elastic - plastic materials and semi brittle materials. Doctor's Thesis. Wroclaw Technical University, Wroclaw 1979.

(3) BOTTANI (C.E.), CAGLIOTI (G.) - Thermal emission: a probe to identify the critical point of the elastoplastic transition. Materials Letters vol.1,December 1982.

[4] KARAMARA (A.), MAJ (M.) - L'etat energetique de la fonte a graphite spheroidal. 53 Congres Mondial de Fonderie, Prague, September 1986.

[5] ASHBY (H.F.) - The deformation non - homogeneus materials. Harvard University. 1969, p.399. 